\title{
Spin squeezing in a driven Bose-Einstein condensate
}

\author{
Stewart D. Jenkins* and T. A. Brian Kennedy ${ }^{\dagger}$ \\ School of Physics, Georgia Institute of Technology, Atlanta, Georgia 30332-0430
}

(Received 1 August 2002; published 30 October 2002)

\begin{abstract}
We consider a collection of atoms prepared in a Bose-Einstein condensate which interact via two-body elastic collisions. A resonant driving field couples two internal states which together constitute an effective spin $1 / 2$ system for each atom, and from which a total spin for the gas can be defined. It is shown that in the limit of strong driving, the system dynamics reduces to a mechanism for spin squeezing similar to that of a recent proposal for undriven condensates. However, we find that the conditions for spatial stability in our driven system are complementary to those of the undriven system. Reasons for this difference, associated with the physics of dressed atom collisions, are discussed along with conditions for preparing and observing the spin squeezed state.
\end{abstract}

DOI: 10.1103/PhysRevA.66.043621

PACS number(s): 03.75.Fi, 03.65.Ud, 03.67.-a

\section{INTRODUCTION}

A system of $N \gg 1$ distinguishable two state quantum systems has a Hilbert space of dimension $2^{N}$. Is it practically possible to prepare arbitrary quantum states, by accessing the recesses of this large space? The preparation of such massively entangled states is a major focus of current research in quantum information physics. By definition, entangled states cannot be represented as product states, or statistical mixtures of product states.

In recent years, a manifestation of a particular form of massive entanglement known as spin squeezing [1] has been of great interest. Such correlated atomic wave functions could be used to enhance the accuracy of population measurements in spectroscopy and also have the potential to be used in atomic clocks [2-4]. Several methods for producing spin squeezed states have been proposed in the last decade [3,5-11]. Experimental realizations of these states have been made by mapping the quantum state of squeezed light onto a gas of cold uncondensed atoms [12], as well as through quantum nondemolition measurements [13,14].

In a recent paper, Sorensen et al. [15] have suggested that an entangled wave function for the internal state of a system of $N$ atoms can be produced by means of low-energy elastic collisions within a vapor prepared in a special initial state. The initial state is a zero-temperature Bose-Einstein condensate (BEC). A short controlled electromagnetic pulse is applied and, at the atomic level, coherently mixes two singleparticle atomic states, labeled by $|+\rangle$ and $|-\rangle$, which form an effective spin $1 / 2$ system. The sum of $N$ such atomic spins defines a resultant $N$-particle spin angular momentum for the gas. The effect of low-energy $s$-wave atomic collisions is to squeeze, hence entangle, the associated $N$-particle spin wave function of the condensate mixture [1]. The special initial condition, and the stability of a common spatial mode for condensates in either of the single-particle atomic states, leads to a squeezing which scales with $N$. This very attractive feature, by contrast with some of the earlier proposals, means

\footnotetext{
*Electronic address: stewart.jenkins@physics.gatech.edu

${ }^{\dagger}$ Electronic address: brian.kennedy@ physics.gatech.edu
}

that entanglement could be enhanced just by increasing the initial condensate particle number.

The stability of the common spatial mode, the so-called "breathe-together" mode is a nontrivial issue. A detailed stability analysis was reported in Ref. [16], and, in the ThomasFermi limit, boils down to a condition on the relative strength of interstate and intrastate $s$-wave atomic collisions, i.e., $g_{+-}<g_{++}, g_{--}$. Here $g_{\mu \nu}$ is proportional to the scattering length for the collision of atoms in the stationary states $|\mu\rangle$ and $|\nu\rangle$, respectively, and is defined precisely later. In short, for a pair of overlapping condensates, interacting only via elastic collisions, a common spatial mode is stable for atomic states which have relatively weak interstate collisions.

In this paper we present a scenario for squeezing the $N$-particle spin wave function in the opposite limit $g_{+-}$ $>\left(g_{++}+g_{--}\right) / 2$, when the interstate collisions are relatively strong. The procedure is similar to that of Ref. [15], and again relies on the existence of a common breathetogether spatial mode, except that the initial BEC is strongly driven by an external field, so that the atomic single-particle states execute resonant Rabi oscillations at a frequency that exceeds other characteristic time scales of the problem. This type of driving between hyperfine ground states was originally demonstrated experimentally in a rubidium BEC by Matthews et al. [17], and has since been used in the creation of vortices $[18,19]$. Spin squeezing is again caused by elastic atomic collisions between condensate atoms which undergo rapid Rabi oscillations, i.e., collisions of dressed atoms. The reasons for the complementary stability criteria are, as will be discussed, a result of the different collision properties of bare atom and dressed atom condensates [20].

The remainder of this paper is organized as follows. In Sec. II we present the basic theoretical model. An analysis of the model follows in Sec. III, where the limit of large Rabi frequency is used to simplify the equations. This section also includes a discussion of the breathe-together mode and its stability, an analytical calculation of the spin squeezing, and a comparison with the physics of Ref. [15]. Following our conclusion in Sec. IV, two appendixes provide further details on the breathe-together mode stability (Appendix A) and the 
collisional interaction energy of bare and dressed condensates (Appendix B).

\section{THEORETICAL MODEL}

We consider an ultracold BEC atomic gas with two single-particle states labeled by $|+\rangle$ and $|-\rangle$, which form a closed two state system. In an alkali atom these states should be hyperfine states belonging to the ground electronic term ${ }^{2} S_{1 / 2}$, although their total angular momenta $F$, and hence their energy, may be different. The possibility of collision induced couplings to other degenerate hyperfine states means that the two state system will not, in general, be closed. This problem may be circumvented if one chooses the two internal states with different $F$ values such that both states have the minimum (or maximum) possible values for $M_{F}$ in their respective manifolds. Doing this will forbid spin-exchange collisions between the two states, thus forming a closed twostate system. Alternatively, if this choice cannot be made, the unwanted degenerate states may be coupled via an off resonant laser field to higher energy levels, thereby raising the energy of the unwanted levels through the ac Stark shift, and making spin-exchange collisions energetically unfavorable [15]. It should be noted that with the former option, it is necessary to use an optical trap [21] to confine the gas; since these states generally have opposite magnetic moments, it would not be possible to simultaneously trap them magnetically. By assuming that a careful choice of the states has been made, we will exclude from our model the influence of other hyperfine states. The role of atomic losses in degrading the squeezing has been discussed in Ref. [15], and we expect similar qualitative features with the current scheme.

BEC atoms in the states $|+\rangle$ and $|-\rangle$ suffer low-energy elastic collisions, while a resonant external electromagnetic field induces transitions between them. The Hamiltonian for the $N$-particle system may be written

$$
\hat{H}(t)=\hat{K}+\hat{V}_{e x t}+\omega_{0} \hat{S}_{z}+\hat{U}_{C}+\hat{H}^{A F}(t),
$$

where $\hat{K}$ is the kinetic energy, $\hat{V}_{\text {ext }}$ is the external trapping potential, $\hat{U}_{C}$ is the collisional interaction energy, and $\hat{H}^{A F}(t)$ is the interaction between the atomic states and the external electromagnetic field. These operators are defined by

$$
\begin{aligned}
\hat{K}=- & \sum_{\mu=+,-} \int d^{3} r \quad \hat{\psi}_{\mu}^{\dagger}(\mathbf{r}) \frac{\hbar^{2} \nabla^{2}}{2 m} \hat{\psi}_{\mu}(\mathbf{r}), \\
\hat{V}_{e x t}= & \int d^{3} r \hat{\psi}_{+}^{\dagger}(\mathbf{r}) V_{+}(\mathbf{r}) \hat{\psi}_{+}(\mathbf{r}) \\
& +\int d^{3} r \hat{\psi}_{-}^{\dagger}(\mathbf{r}) V_{-}(\mathbf{r}) \hat{\psi}_{-}(\mathbf{r}), \\
\hat{H}^{A F}(t)= & -\frac{\hbar}{2}\left(\int d^{3} r \hat{\psi}_{+}^{\dagger}(\mathbf{r}) \kappa(\mathbf{r}) e^{-i \phi(\mathbf{r})-i \omega_{L} t}\right. \\
& \left.\times \hat{\psi}_{-}(\mathbf{r})+\text { H.c. }\right),
\end{aligned}
$$

where $\hat{\psi}_{\mu}(\mathbf{r})$ and $\hat{\psi}_{\mu}^{\dagger}(\mathbf{r})$ are boson field annihilation and creation operators for the bare atomic states, $\kappa(\mathbf{r})$ and $\phi(\mathbf{r})$ are the effective Rabi frequency and phase of the external driving field, respectively, and $V_{ \pm}(\mathbf{r})$ are the external trapping potentials for the atomic states $| \pm\rangle$. For transitions between two hyperfine states separated by a microwave frequency, the wavelength can be assumed large with respect to the vapor, and we can take the Rabi frequency and phase to be constant throughout the sample, i.e., $\phi(\mathbf{r})=0$ and $\kappa(\mathbf{r})=\kappa$. The transition frequency between the states is $\omega_{0}$ and the spin operator $\hat{S}_{z}$ is defined in terms of the Pauli spin matrix $\sigma_{z}$,

$$
\hat{S}_{z}=\frac{\hbar}{2} \sum_{\mu, \nu=+,-} \int d^{3} r \hat{\psi}_{\mu}^{\dagger}(\mathbf{r})\left(\sigma_{z}\right)_{\mu \nu} \hat{\psi}_{\nu}(\mathbf{r}) .
$$

We similarly define $\hat{S}_{x}$ and $\hat{S}_{y}$, by replacing $\sigma_{z}$ with $\sigma_{x}$ and $\sigma_{y}$, respectively.

The two-body collisional interaction potential may be written

$$
\hat{U}_{C}=\frac{1}{2} \sum_{\mu, \nu= \pm} g_{\mu \nu} \int d^{3} r \quad \hat{\psi}_{\mu}^{\dagger}(\mathbf{r}) \hat{\psi}_{\nu}^{\dagger}(\mathbf{r}) \hat{\psi}_{\nu}(\mathbf{r}) \hat{\psi}_{\mu}(\mathbf{r}),
$$

where the collision coefficients $g_{\mu \nu}=4 \pi^{2} \hbar^{2} a_{\mu \nu} / m$ with $a_{\mu \nu}$ the scattering length between atoms in the internal states $\mu$ and $\nu$. Note that aside from the atomic energy and Rabi coupling terms in the Hamiltonian, this is exactly the same Hamiltonian discussed by Sorensen et al. [15].

\section{ANALYSIS OF THE MODEL}

\section{A. Limit of large Rabi frequency}

We consider the limit that the Rabi frequency $\kappa$ is the largest frequency in the problem. The analysis then proceeds by means of two unitary transformations [22]. The first is generated by $\hat{U}(t)=\exp \left(-i \omega_{L} \hat{S}_{z} / \hbar\right)$, and yields a time dependent Schrodinger equation with a time independent Hamiltonian $\hat{H}^{\prime \prime}$. The latter is identical to the Hamiltonian $\hat{H}(t)$ with the substitutions $\hat{H}^{A F}(t) \rightarrow \hat{H}^{A F}(0)=-\kappa \hat{S}_{x}$ and $\omega_{0}$ $\rightarrow \Delta=\omega_{0}-\omega_{L}$. Assuming atomic resonance, $\Delta=0$, we may write $\hat{H}^{\prime \prime}$ as

$$
\hat{H}^{\prime \prime}=\hat{K}+\hat{V}_{e x t}+\hat{U}_{C}-\kappa \hat{S}_{x}
$$

A second unitary transformation is applied with $\hat{W}(t)$ $=\exp \left(i \kappa \hat{S}_{x} / \hbar\right)$, which yields the Schrödinger equation $i \hbar(\partial / \partial t)|\psi(t)\rangle=\hat{H}^{\prime}(t)|\psi(t)\rangle$ with $\hat{H}^{\prime}(t)=\hat{K}+\hat{W}^{\dagger}(t)\left(\hat{V}_{e x t}\right.$ $\left.+\hat{U}_{C}\right) \hat{W}(t)$. Here, $|\psi(t)\rangle$ is the state vector in the rotating frame, and is related to the Schrödinger picture state vector in the laboratory frame $|\Psi(t)\rangle$, by $|\Psi(t)\rangle$ $=\hat{U}(t) \hat{W}(t)|\psi(t)\rangle$.

Transformed to the rotating frame the one-body potential becomes 


$$
\begin{aligned}
\hat{W}^{\dagger} \hat{V}_{e x t} \hat{W}= & \sum_{\mu=+,-} \int d^{3} r \hat{\psi}_{\mu}^{\dagger}(\mathbf{r}) \bar{V}(\mathbf{r}) \hat{\psi}_{\mu}(\mathbf{r}) \\
& +\cos \kappa t \int d^{3} r\left[\hat{\psi}_{+}^{\dagger}(\mathbf{r}) V_{d}(\mathbf{r}) \hat{\psi}_{+}(\mathbf{r})\right. \\
& \left.-\hat{\psi}_{-}^{\dagger}(\mathbf{r}) V_{d}(\mathbf{r}) \hat{\psi}_{-}(\mathbf{r})\right] \\
& +i \sin \kappa t \int d^{3} r\left[\hat{\psi}_{+}^{\dagger}(\mathbf{r}) V_{d}(\mathbf{r}) \hat{\psi}_{-}(\mathbf{r})\right. \\
& \left.-\hat{\psi}_{-}^{\dagger}(\mathbf{r}) V_{d}(\mathbf{r}) \hat{\psi}_{+}(\mathbf{r})\right],
\end{aligned}
$$

where $\quad \bar{V}(\mathbf{r})=\frac{1}{2}\left[V_{+}(\mathbf{r})+V_{-}(\mathbf{r})\right] \quad$ and $\quad V_{d}(\mathbf{r})=\frac{1}{2}\left[V_{+}(\mathbf{r})\right.$ $\left.-V_{-}(\mathbf{r})\right]$. This expression is formally in agreement with the mean-field theory of Williams et al. [22]. The two-body interaction potentials transform as

$$
\begin{aligned}
\hat{W}^{\dagger} \hat{U}_{C} \hat{W}= & \bar{g} \int d^{3} r \hat{T}_{0}^{(0)}(\mathbf{r})+g^{\prime} \int d^{3} r\left[\hat{T}_{z}^{(1)}(\mathbf{r}) \cos \kappa t\right. \\
& \left.+\hat{T}_{y}^{(1)}(\mathbf{r}) \sin \kappa t\right]-g \int d^{3} r\left[e^{2 i \kappa t} \hat{T}_{-2}^{(2)}(\mathbf{r})\right. \\
& \left.+e^{-2 i \kappa t} \hat{T}_{2}^{(2)}(\mathbf{r})\right] \\
& +g \int d^{3} r \hat{\psi}_{x+}^{\dagger}(\mathbf{r}) \hat{\psi}_{x-}^{\dagger}(\mathbf{r}) \hat{\psi}_{x-}(\mathbf{r}) \hat{\psi}_{x+}(\mathbf{r}),
\end{aligned}
$$

where $\hat{\psi}_{x+}^{\dagger}(\mathbf{r})\left[\hat{\psi}_{x-}^{\dagger}(\mathbf{r})\right]$ are bosonic field creation operators for a spin one-half oriented in the positive [negative] $x$ direction, i.e., $\hat{\psi}_{x+}^{\dagger}(\mathbf{r})=\left[\hat{\psi}_{+}^{\dagger}(\mathbf{r})+\hat{\psi}_{-}^{\dagger}(\mathbf{r})\right] / \sqrt{2}$ and $\hat{\psi}_{x-}^{\dagger}(\mathbf{r})=$ $-i\left[\hat{\psi}_{+}^{\dagger}(\mathbf{r})-\hat{\psi}_{-}^{\dagger}(\mathbf{r})\right] / \sqrt{2}[26]$. Alternatively, it is useful to regard these as field operators for dressed atomic singleparticle states, arising as a result of the Rabi coupling of the bare atom to the external electromagnetic field [20].

The collision coupling coefficients are given by

$$
\begin{gathered}
\bar{g}=\frac{1}{4} \sum_{\mu, \nu=+,-} g_{\mu \nu}, \\
g^{\prime}=\frac{1}{4}\left(g_{++}-g_{--}\right), \\
g=\frac{1}{4}\left(g_{++}+g_{--}-2 g_{+-}\right) .
\end{gathered}
$$

We have also defined the collision kernels $\hat{T}_{q}^{(k)}(\mathbf{r})$ as follows:

$$
\begin{aligned}
\hat{T}_{0}^{(0)}(\mathbf{r}) & =\sum_{\mu, \nu= \pm} \hat{\psi}_{\mu}^{\dagger}(\mathbf{r}) \hat{\psi}_{\nu}^{\dagger}(\mathbf{r}) \hat{\psi}_{\nu}(\mathbf{r}) \hat{\psi}_{\mu}(\mathbf{r}), \\
\hat{T}_{ \pm 1}^{(1)}(\mathbf{r})= & \mp \frac{1}{\sqrt{2}}\left[\hat{\psi}_{x+}^{\dagger}(\mathbf{r}) \hat{\psi}_{x \pm}^{\dagger}(\mathbf{r}) \hat{\psi}_{x \mp}(\mathbf{r}) \hat{\psi}_{x+}(\mathbf{r})\right. \\
& \left.+\hat{\psi}_{x-}^{\dagger}(\mathbf{r}) \hat{\psi}_{x \pm}^{\dagger}(\mathbf{r}) \hat{\psi}_{x \mp}(\mathbf{r}) \hat{\psi}_{x-}(\mathbf{r})\right],
\end{aligned}
$$

$$
\hat{T}_{ \pm 2}^{(2)}=\frac{1}{2} \hat{\psi}_{x \pm}^{\dagger}(\mathbf{r}) \hat{\psi}_{x \pm}^{\dagger}(\mathbf{r}) \hat{\psi}_{x \mp}(\mathbf{r}) \hat{\psi}_{x \mp}(\mathbf{r})
$$

and $\mp \sqrt{2} \hat{T}_{ \pm 1}^{(1)}(\mathbf{r})=\hat{T}_{y}^{(1)}(\mathbf{r}) \pm i \hat{T}_{z}^{(1)}(\mathbf{r})$.

The notation $\hat{T}_{q}^{(k)}$ indicates the $q$ component of a rank $k$ spherical tensor with respect to the effective spin, as defined by the commutation relations

$$
\begin{gathered}
{\left[\hat{S}_{x}, \hat{T}_{q}^{(k)}(\mathbf{r})\right]=\hbar q \hat{T}_{q}^{(k)}(\mathbf{r}),} \\
{\left[\hat{S}_{y} \pm i \hat{S}_{z}, \hat{T}_{q}^{(k)}(\mathbf{r})\right]=\hbar \sqrt{(k \mp q)(k \pm q+1)} \hat{T}_{q \pm 1}^{(k)}(\mathbf{r}) .}
\end{gathered}
$$

Rewriting the collision interaction in terms of these spherical tensors is useful for the purpose of calculating the unitary transformation, since it follows from their definition that

$$
\hat{W}^{\dagger}(t) \hat{T}_{q}^{(k)}(\mathbf{r}) \hat{W}(t)=e^{-i q \kappa t} \hat{T}_{q}^{(k)}(\mathbf{r}) .
$$

The one collision kernel that has not been explicitly defined in terms of tensors, is a linear combination of tensors of rank 0 and 2, each with component $q=0$. As a consequence, it is unaffected by the second unitary transformation, and thus we leave it in the explicit form given.

We are interested in the limit of large Rabi frequency compared to any other characteristic frequency. In this limit we make a form of rotating wave approximation and cycle average the rotating frame Hamiltonian $\hat{H}^{\prime}(t)$ over the Rabi period to get

$$
\begin{aligned}
\hat{H}_{R W A}^{\prime}= & \hat{K}+\sum_{\mu= \pm} \int d^{3} r \hat{\psi}_{\mu}^{\dagger}(\mathbf{r}) \bar{V}(\mathbf{r}) \hat{\psi}_{\mu}(\mathbf{r}) \\
& +\frac{1}{2} \bar{g} \sum_{\mu, \nu= \pm} \int d^{3} r \hat{\psi}_{\mu}^{\dagger}(\mathbf{r}) \hat{\psi}_{\nu}^{\dagger}(\mathbf{r}) \hat{\psi}_{\nu}(\mathbf{r}) \hat{\psi}_{\mu}(\mathbf{r}) \\
& +g \int d^{3} r \hat{\psi}_{x+}^{\dagger}(\mathbf{r}) \hat{\psi}_{x-}^{\dagger}(\mathbf{r}) \hat{\psi}_{x-}(\mathbf{r}) \hat{\psi}_{x+}(\mathbf{r}),
\end{aligned}
$$

in which all collision tensors with $q \neq 0$ have disappeared. In the limit of strong driving the vapor experiences only the average external potential $\bar{V}(\mathbf{r})$ of the two strongly coupled single-particle states [22]. In a far off-resonance optical trap [21], unlike a magnetic trap, it is reasonable to assume that the latter potentials are identical, and thus equal to $\bar{V}(\mathbf{r})$.

\section{B. Single-mode approximation: the breathe-together mode}

In the rotating frame the Hamiltonian $H_{R W A}^{\prime}$ is independent of the driving field. This enables us to make use of the analysis of Ref. [16], and assume the existence of a stable single common "breathe-together" mode $\bar{\phi}(\mathbf{r}, t)$. Perhaps surprisingly, the condition for stability of this mode, $g_{+-}$ $>\left(g_{++}+g_{--}\right) / 2$, is opposite to that required for its stability in the BEC squeezing scheme of Ref. [15]. In the latter scheme squeezing results from the collisional interaction of two bare atomic condensates without any Rabi coupling.

A detailed discussion of the stability condition is given in Appendix A. A qualitative argument based on a calculation 
of the mean interaction energy of two condensates in which the macroscopically occupied single-particle states are, respectively, bare atomic states $E_{i n t}^{b}$ and dressed atomic states $E_{i n t}^{d}$, is revealing (Appendix B). These energies are given by

$$
E_{\text {int }}^{b}=g_{+-} \int d^{3} r \rho_{+}(\mathbf{r}) \rho_{-}(\mathbf{r}),
$$

where $\rho_{ \pm}(\mathbf{r}) \equiv N_{ \pm}\left|\phi_{ \pm}(\mathbf{r})\right|^{2}$ are the spatial densities of the bare states, and

$$
E_{\text {int }}^{d}=\frac{1}{2}\left(g_{++}+g_{--}\right) \int d^{3} r \rho_{x+}(\mathbf{r}) \rho_{x-}(\mathbf{r}),
$$

where $\rho_{x \pm}(\mathbf{r}) \equiv N_{x \pm}\left|\phi_{x \pm}(\mathbf{r})\right|^{2}$ are the spatial densities of the dressed states. The interaction energy is proportional to the overlap integral of the corresponding condensate spatial density profiles, with the expected prefactor $g_{+-}$in the bare atom case, but with a prefactor $\left(g_{++}+g_{--}\right) / 2$ for dressed condensates. Indeed the coefficient $g_{+-}$only contributes to the self-interaction energy of dressed condensates. These unusual features of elastic collisions in dressed condensates are reflected in the different stability criterion for the breathetogether mode.

Assuming the atomic states are chosen such that the stability condition for the breathe-together mode is satisfied, we may define annihilation operators for this mode by $[20,23,24]$

$$
\hat{a}_{x \pm}(t)=\int d^{3} r \bar{\phi}^{*}(\mathbf{r}, t) \hat{\psi}_{x \pm}(\mathbf{r}),
$$

with corresponding definitions for creation operators, while the number operator $\hat{a}_{x \pm}^{\dagger}(t) \hat{a}_{x \pm}(t)=\hat{a}_{x \pm}^{\dagger} \hat{a}_{x \pm}$ is time independent.

Hence, in $\hat{H}_{R W A}^{\prime}$ we replace the field operators $\hat{\psi}_{x+}(\mathbf{r})$ $\rightarrow \bar{\phi}(\mathbf{r}, t) \hat{a}_{x+}(t), \hat{\psi}_{x-}(\mathbf{r}) \rightarrow \bar{\phi}(\mathbf{r}, t) \hat{a}_{x-}(t)$ to give

$$
\begin{aligned}
\hat{H}_{R W A}^{\prime} & \rightarrow N E(t)+\hbar \Omega(t) \hat{a}_{x+}^{\dagger} \hat{a}_{x-}^{\dagger} \hat{a}_{x-} \hat{a}_{x+} \\
& =N E(t)-\hbar \Omega(t)\left(\frac{\hat{S}_{x}^{2}}{\hbar^{2}}-\frac{1}{4} N^{2}\right),
\end{aligned}
$$

where

$$
\begin{aligned}
E= & \int d^{3} r \bar{\phi}^{*}(\mathbf{r}, t)(K+\bar{V}) \bar{\phi}(\mathbf{r}, t) \\
& +\frac{1}{2} \bar{g}\left(\int d^{3} r|\bar{\phi}(\mathbf{r}, t)|^{4}\right)(N-1)
\end{aligned}
$$

and

$$
\hbar \Omega(t)=g\left(\int d^{3} r|\bar{\phi}(\mathbf{r}, t)|^{4}\right) .
$$

Thus in the breathe-together mode approximation $H_{R W A}^{\prime}$ reduces to the one-axis twist Hamiltonian originally discussed in the context of spin squeezing by Kitagawa and Ueda [1]. The nomenclature arises since $\hat{S}_{x}$ generates a rotation about the $x$ axis, and $\hat{S}_{x}^{2}$ generates a rotation in which the eigenstates of $\hat{S}_{x}$ are rotated in opposite directions.

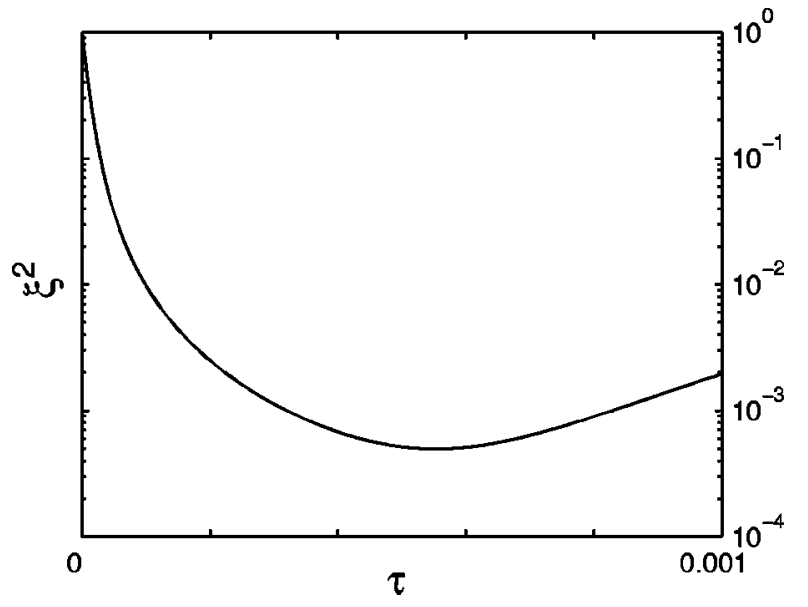

FIG. 1. The squeezing parameter $\xi^{2}$ as a function of time, $\tau$ $=\int_{0}^{t} \Omega\left(t^{\prime}\right) d t^{\prime}$, for the evolution of the system under the Hamiltonian $-\Omega(t) \hat{S}_{x}^{2} / \hbar$. The initial state has all of the particles in the single-particle state $|-\rangle$, and $N=10^{5}$.

The degree of spin squeezing that results from time evolution under this Hamiltonian can be quantified by the parameter $\xi^{2}=\inf \left\{N\left(\Delta \hat{S}_{\perp}\right)^{2} /|\langle\hat{\mathbf{S}}\rangle|^{2}: \hat{S}_{\perp}=\hat{\mathbf{S}} \cdot \mathbf{n}\right.$, where $\left.\mathbf{n} \perp\langle\hat{\mathbf{S}}\rangle\right\}$. This definition for the degree of entanglement is consistent with those discussed by Wineland et al. $[3,4]$ and Sorensen et al. [15]. It has been shown that if $\xi^{2}<1$, then the condensate exhibits massive entanglement and spin squeezing [15]. It can also be demonstrated that we attain maximal squeezing when our initial state vector is a coherent spin state (as defined by Kitagawa and Ueda [1]) whose mean spin vector is perpendicular to the axis about which the one axis twisting occurs, in this case the $x$ axis. A convenient realization of this initial condition would be a zero-temperature BEC with all atoms in the internal state, $|-\rangle$.

With the coherent state initial condition, the squeezing parameter as a function of time can be computed analytically [1], and is given by

$$
\xi^{2}(t)=\frac{\left\{\left[1+\frac{1}{4}(N-1) A(t)\right]-\frac{1}{4}(N-1) \sqrt{A(t)^{2}+B(t)^{2}}\right\}}{\cos ^{2 N-2}\left(\int_{0}^{t} \Omega\left(t^{\prime}\right) d t^{\prime}\right)},
$$

where $\quad A(t)=1-\cos ^{N-2}\left[2 \int_{0}^{t} \Omega\left(t^{\prime}\right) d t^{\prime}\right], \quad$ and $\quad B(t)=$ $-4 \sin \left[\int_{0}^{t} \Omega\left(t^{\prime}\right) d t^{\prime}\right] \cos ^{N-2}\left[\int_{0}^{t} \Omega\left(t^{\prime}\right) d t^{\prime}\right]$ (Fig. 1). For large $N$, it has been shown that the minimum attainable squeezing parameter scales as $\xi^{2} \sim N^{-2 / 3}[1]$.

For spin squeezing to occur the collision coefficient $g$ must be nonzero. Furthermore, for our approximations to be valid, the characteristic frequency $\Omega$ must be small compared to the Rabi frequency $\kappa$. To illustrate the time scales, we give some estimates of the characteristic parameters. We assume, for simplicity, that the vapor is trapped in a spherically symmetric potential $V(r)=V_{-}(r)=V_{+}(r)$ $=\frac{1}{2} m \omega_{\text {trap }}^{2} r^{2}$. The initial wave function $\phi_{0}(\mathbf{r})$ can be determined using the Thomas-Fermi approximation to the time 
independent Gross-Pitaevski equation [25]. Using this procedure we numerically determine the characteristic frequency $\Omega$ to be

$$
\begin{aligned}
\Omega\left[\mathrm{s}^{-1}\right]= & 1.4 \times 10^{-4} m\left(a_{++}+a_{--}-2 a_{+-}\right) \\
& \times\left(\omega_{\text {trap }}\left[\mathrm{s}^{-1}\right]\right)^{6 / 5}\left(\frac{a_{\text {init }}}{N}\right)^{3 / 5},
\end{aligned}
$$

where the scattering lengths are given in Bohr radii, and the atomic mass $m$ is measured in atomic mass units. The scattering length $a_{\text {init }}$ corresponds to the initial condensate, prior to the application of the external electromagnetic field. For example, if the system condenses in the single-particle state $|-\rangle$, then $a_{\text {init }}=a_{--}$.

We further note, using the results of Ref. [1], that the time taken to attain the minimum squeezing parameter is given by

$$
t_{\min }[\mathrm{s}] \approx \frac{3.7 \times 10^{4}}{a_{\text {init }}^{3 / 5}} \frac{m^{-1 / 5}\left(\omega_{\text {trap }}\left[\mathrm{s}^{-1}\right]\right)^{-6 / 5} N^{-1 / 15}}{\left(a_{++}+a_{--}-2 a_{+-}\right)},
$$

which for $\omega_{\text {trap }} / 2 \pi \approx 1 \mathrm{kHz}$, is typically a fraction of a second, virtually independent of $N$ for practical purposes.

\section{Discussion of BEC spin squeezing scenarios}

In the scheme of Ref. [15], spin squeezing is produced by coherent elastic collisions in a BEC mixture of atoms which individually constitute effective spin one-half bosons. A fast $\pi / 2$ pulse is applied to the condensate with all atoms initially prepared in the internal state $|-\rangle$ (for ease of comparison we will use as close a notation as is possible to that employed in the rest of our paper). The $\pi / 2$ pulse produces a bare atom condensate mixture with all of the "spins" oriented in the positive $x$ direction. Elastic collisions then act to squeeze the initial coherent spin state. By contrast, in our scheme, elastic collisions take place during the resonant Rabi oscillations induced by a strong applied field, squeezing the dressed atom condensate mixture.

The Hamiltonian of Ref. [15] is given in our notation by $\hat{H}_{\text {sor }} \equiv \hat{H}(t)-\hat{H}^{A F}(t)\left(\right.$ with $\left.\omega_{0} \equiv 0\right)$,

$$
\begin{aligned}
\hat{H}_{s o r}= & \hat{K}+\hat{V}+\frac{1}{2} \sum_{\mu} g_{\mu \mu} \int d^{3} r \hat{\psi}_{\mu}^{\dagger}(\mathbf{r}) \hat{\psi}_{\mu}^{\dagger}(\mathbf{r}) \hat{\psi}_{\mu}(\mathbf{r}) \hat{\psi}_{\mu}(\mathbf{r})+g_{+-} \int d^{3} r \hat{\psi}_{+}^{\dagger}(\mathbf{r}) \hat{\psi}_{-}^{\dagger}(\mathbf{r}) \hat{\psi}_{-}(\mathbf{r}) \hat{\psi}_{+}(\mathbf{r}) \\
= & \hat{K}+\hat{V}+\frac{1}{4}\left(g_{++}+g_{--}\right) \sum_{\mu, \nu} \int d^{3} r \hat{\psi}_{\mu}^{\dagger}(\mathbf{r}) \hat{\psi}_{\nu}^{\dagger}(\mathbf{r}) \hat{\psi}_{\nu}(\mathbf{r}) \hat{\psi}_{\mu}+\frac{1}{4}\left(g_{++} g_{--}\right) \\
& \times \int d^{3} r \hat{\psi}_{+}^{\dagger}(\mathbf{r}) \hat{\psi}_{+}^{\dagger}(\mathbf{r}) \hat{\psi}_{+}(\mathbf{r}) \hat{\psi}_{+}(\mathbf{r})-\frac{1}{4}\left(g_{++}-g_{--}\right) \int d^{3} r \hat{\psi}_{-}^{\dagger}(\mathbf{r}) \hat{\psi}_{-}^{\dagger}(\mathbf{r}) \hat{\psi}_{-}(\mathbf{r}) \hat{\psi}_{-}(\mathbf{r}) \\
& -2 g \int d^{3} r \hat{\psi}_{+}^{\dagger}(\mathbf{r}) \hat{\psi}_{-}^{\dagger}(\mathbf{r}) \hat{\psi}_{-}(\mathbf{r}) \hat{\psi}_{+}(\mathbf{r}) .
\end{aligned}
$$

For $g_{++}=g_{--}$, which is satisfied in the case of sodium, $\hat{H}_{\text {sor }}$ reduces to a form very similar to $\hat{H}_{R W A}^{\prime}$ in Eq. (18). The last term in $\hat{H}_{\text {sor }}$, causes spin squeezing by means of a $z$ axis twist. The operator $\hat{S}_{z}$ is diagonal for states with a definite occupancy of the single-particle states $| \pm\rangle$. With $\hat{H}_{R W A}^{\prime}$, the wave function in the rotating frame is squeezed by an $x$-axis twist; $\hat{S}_{x}$ is diagonal for states with definite occupancy of the single-particle dressed states $|x \pm\rangle$. However, in both cases squeezing relies on an effective single-mode approximation. As discussed in the last subsection, and in more detail in Appendix A, the stability criteria for the existence of the breathe-together mode on the intraparticle and interparticle scattering lengths are opposite inequalities in the two scenarios, i.e., $g_{+-}<g_{++}, g_{--}$for bare condensates, and $g_{+-}>\left(g_{++}+g_{--}\right) / 2$ for dressed condensates. The two scenarios are thus complementary rather than alternatives. In the single-mode approximation, $\hat{H}_{\text {sor }}$ contains the $z$-axis twist operator $2 \Omega \hat{S}_{z}^{2} / \hbar$, while $\hat{H}_{R W A}^{\prime}$ contains the $x$-axis twist
$-\Omega \hat{S}_{x}^{2} / \hbar$. The factor two difference arises because we average over fast Rabi oscillations in the derivation of $\hat{H}_{R W A}^{\prime}$. In Ref. [15], factors such as multiple modes and atomic losses, which reduce or destroy squeezing are analyzed. We will not discuss these further, as we expect their effect to be qualitatively similar here.

Finally we discuss the spin dynamics from the viewpoint of the laboratory frame. In Ref. [15], squeezing occurs as a result of free evolution of a condensate in which the constituent atomic "spins" are all oriented in the positive $x$ direction. As the condensate evolves, the orientation of the mean spin vector will not change at all if $g_{++}=g_{--}$and the motional degrees of freedom are neglected. Squeezing could be detected in a component transverse to the nonzero average spin component $\left\langle\Psi(t)\left|\hat{S}_{x}\right| \Psi(t)\right\rangle \neq 0$. In our approach, the external field drives rapid Rabi oscillations in the populations of the $|+\rangle$ and $|-\rangle$ states. If we examine the evolution of the state vector in the rotating frame $|\psi(t)\rangle$, we see that the 
mean spin vector maintains the same orientation, along the negative $z$ axis, so that $\left\langle\psi(t)\left|\hat{S}_{z}\right| \psi(t)\right\rangle<0$ and $\left\langle\psi(t)\left|\hat{S}_{x, y}\right| \psi(t)\right\rangle=0$, although its magnitude shrinks with time. In the laboratory frame, relevant spin observables should be computed with the lab frame state vector $|\Psi(t)\rangle$ $=\hat{U}(t) \hat{W}(t)|\psi(t)\rangle$ and the similarly transformed spin operator $\hat{\mathbf{S}}_{R}(t)=U(t) W(t) \hat{\mathbf{S}} W^{\dagger}(t) U^{\dagger}(t)$. The latter can be written explicitly as

$$
\left(\begin{array}{c}
\hat{S}_{x R}(t) \\
\hat{S}_{y R}(t) \\
\hat{S}_{z R}(t)
\end{array}\right)=\left(\begin{array}{ccc}
1 & 0 & 0 \\
0 & \cos \kappa t & \sin \kappa t \\
0 & -\sin \kappa t & \cos \kappa t
\end{array}\right)\left(\begin{array}{ccc}
\cos \omega_{L} t & \sin \omega_{L} t & 0 \\
-\sin \omega_{L} t & \cos \omega_{L} t & 0 \\
0 & 0 & 1
\end{array}\right)\left(\begin{array}{c}
\hat{S}_{x} \\
\hat{S}_{y} \\
\hat{S}_{z}
\end{array}\right)
$$

In terms of the laboratory frame Schrödinger picture state vector $|\Psi(t)\rangle$, we have, for example, $\langle\psi(t)|\hat{\mathbf{S}}| \psi(t)\rangle$ $=\left\langle\Psi(t)\left|\hat{\mathbf{S}}_{R}(t)\right| \Psi(t)\right\rangle$.

The system is subject to two rotations: one about the $z$ axis at a frequency $\omega_{L}$, and another rotation about the negative $x$ axis at frequency $\kappa$. In the lab frame, one would see the mean spin vector rotating about the negative $x$ axis at frequency $\kappa$; this behavior is then superimposed on a rotation about the $z$ axis at frequency $\omega_{L}$. The spin squeezing manifests itself as a modulation on the rotational motion of the mean spin, and occurs along axes that are perpendicular to this vector.

The spin squeezing could, in principle, be observed using a method similar to that described by Wineland et al. [4]. For the purpose of measuring the spin components orthogonal to the mean spin, it is necessary to have the mean spin oriented in a known direction, say along the $z$ axis. Since the direction of the mean spin vector oscillates rapidly, making such a measurement may be difficult at an arbitrary time. This obstacle may be overcome in the following way. Near the time when maximum squeezing is attained, $t_{\min }$, a time $T$ is chosen such that $\kappa T=2 n \pi$ for some integer $n$. The coupling field would then be turned off leaving the Schrödinger picture and rotating frame state vectors related by $|\Psi(T)\rangle=\hat{U}(T) \hat{W}(T)|\psi(T)\rangle=\hat{U}(T) \exp \left[i \hat{S}_{x}(2 n \pi / \hbar)\right]|\psi(T)\rangle$ $=\hat{U}(T)|\psi(T)\rangle$. Since the mean spin in the rotating frame is oriented along the $-z$ axis for all times [1], and $\hat{U}(T)$ is nothing more than a rotation of $\omega_{0} T$ about the $z$ axis, the mean spin as measured in the laboratory will be oriented along the $-z$ direction. The same Schrödinger state vector $|\Psi(T)\rangle$ may also be obtained by a variation of Ramsey spectroscopy, by employing two resonant pulses, each of time duration $T / 2$, and with Rabi frequencies $\kappa$ and $-\kappa$, respectively. The net effect is that $\hat{W}(T)=\exp \left(i \kappa T \hat{S}_{x} / 2 \hbar\right) \exp$ $\left(-i \kappa T \hat{S}_{x} / 2 \hbar\right)=\hat{1}$. We have already shown that the state vector in the rotating frame, $|\psi(T)\rangle$, is squeezed independent of $\kappa$, since $\hat{H}_{R W A}^{\prime}$ is independent of $\kappa$.

Once the final state has been produced, the standard deviation of the spin along an arbitrary axis perpendicular to the mean spin vector is measured $[4,15]$. To do this, the system should be rotated about the $\hat{\mathbf{z}}$ axis by an appropriate amount, and then a $\pi / 2$ rotation about the $\hat{\mathbf{y}}$ axis should be applied. The population of either the $|+\rangle$ or $|-\rangle$ state is then measured, thereby also measuring $\hat{S}_{z}$. A sub-shot-noise measurement of the projection noise indicates spin squeezing $[3,4]$.

\section{CONCLUSION}

We have considered the possibility of spin squeezing in a resonantly driven mixture of Bose-Einstein condensates. In the limit that the Rabi frequency is the largest frequency scale in the problem, we have shown that elastic collisions squeeze quantum fluctuations of the spin component transverse to the average spin vector, according to the single-axis twist mechanism of Ref. [1].

We have further shown that the proposed scheme is complementary to the proposal of Sorensen et al. [15] for spin squeezing of a BEC. While both scenarios depend on the existence of a mutually stable spatial mode for the interacting condensates, the stability criteria are mutually exclusive: $g_{+-}<g_{++}, g_{--}$for Ref. [15], but $g_{+-}>\left(g_{++}\right.$ $\left.+g_{--}\right) / 2$ here. The new stability criterion was attributed to the different collision properties of bare and dressed condensate mixtures, and is discussed in the appendixes. The present proposal is thus limited to atoms with relatively strong interstate collisions, just as Ref. [15] is limited to those with strong intrastate collisions.

\section{ACKNOWLEDGMENTS}

We wish to thank M. Chapman, C. Raman, and A. Kuzmich for useful discussions, and the NSF, Grant No. 9803180, and NSA, under Grant No. ARO DAA55-98-10370, for support. One of us (T.A.B.K.) would also like to thank the Aspen Center for its hospitality.

\section{APPENDIX A: STABILITY OF THE BREATHE-TOGETHER MODE}

We begin by rewriting the rotating wave Hamiltonian $H_{R W A}^{\prime}$ in the form 


$$
\begin{aligned}
\hat{H}_{R W A}^{\prime}= & \hat{K}+\sum_{\mu=a, b} \int d^{3} r \quad \hat{\psi}_{\mu}^{\dagger}(\mathbf{r}) \bar{V}(\mathbf{r}) \hat{\psi}_{\mu}(\mathbf{r}) \\
& +\frac{1}{2} \sum_{\mu=a, b} g_{\mu \mu} \int d^{3} r \hat{\psi}_{\mu}^{\dagger}(\mathbf{r}) \hat{\psi}_{\mu}^{\dagger}(\mathbf{r}) \hat{\psi}_{\mu}(\mathbf{r}) \hat{\psi}_{\mu}(\mathbf{r}) \\
& +g_{a b} \int d^{3} r \quad \hat{\psi}_{a}^{\dagger}(\mathbf{r}) \hat{\psi}_{b}^{\dagger}(\mathbf{r}) \hat{\psi}_{b}(\mathbf{r}) \hat{\psi}_{a}(\mathbf{r}),
\end{aligned}
$$

where, for ease of our subsequent comparison, we use the notation $|x+\rangle=|a\rangle$ and $|x-\rangle=|b\rangle$ to label the singleparticle dressed states. The effective scattering coefficients are given by

$$
\begin{gathered}
g_{a a}=g_{b b}=\frac{1}{4}\left(g_{++}+g_{--}+2 g_{+-}\right), \\
g_{a b}=\frac{1}{2}\left(g_{++}+g_{--}\right) .
\end{gathered}
$$

We can now adapt the results of Sinatra and Castin [16], to identify an effective single mode or "breathe-together" solution. These authors considered the collisional interaction of a pair of condensates with a Hamiltonian identical in form to Eq. (A1), but with $a$ and $b$ corresponding to bare atomic single-particle states, rather than dressed states. We briefly summarize the relevant theory in order to make the arguments clear.

By preparing an initial condensate in a self-consistent mode $\phi_{0}(\mathbf{r})$ and in the bare atomic state $|-\rangle=(|a\rangle$ $-i|b\rangle) / \sqrt{2} \equiv c_{a}|a\rangle+c_{b}|b\rangle[26]$, we have the corresponding $N$ particle initial state in the rotating frame

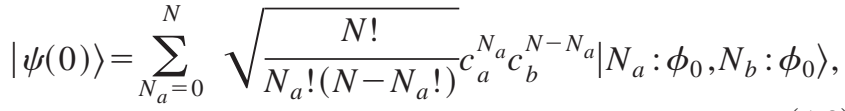

where $N_{a}$ and $N_{b}$ are the occupancies of the dressed states $a$ and $b$. The time evolution of this state according to $H_{R W A}^{\prime}$ is given by

$$
\begin{aligned}
|\psi(t)\rangle= & \sum_{N_{a}=0}^{N} \sqrt{\frac{N !}{N_{a} !\left(N-N_{a}\right) !}} c_{a}^{N_{a}} c_{b}^{N-N_{a}} e^{-i A\left(N_{a}, N_{b} ; t\right)} \\
& \times\left|N_{a}: \phi_{a}\left(N_{a}, N_{b} ; t\right), N_{b}: \phi_{b}\left(N_{a}, N_{b} ; t\right)\right\rangle, \quad
\end{aligned}
$$

where $\phi_{a}(\mathbf{r}, 0)=\phi_{b}(\mathbf{r}, 0)=\phi_{0}(\mathbf{r})$ for all $N_{a}$. The dressed state mode functions $\phi_{a, b}\left(N_{a}, N_{b} ; \mathbf{r}, t\right) \equiv \phi_{a, b}(\mathbf{r}, t)$ satisfy the coupled Gross-Pitaevskii equations,

$$
\begin{aligned}
\frac{\partial \phi_{\mu}(\mathbf{r}, t)}{\partial t}= & \frac{1}{i \hbar}\left(-\frac{\hbar^{2}}{2 m} \nabla^{2}+\bar{V}(\mathbf{r})+\sum_{\nu=a, b} g_{\mu \nu} N_{\nu}\left|\phi_{\nu}(\mathbf{r}, t)\right|^{2}\right) \\
& \times \phi_{\mu}(\mathbf{r}, t)
\end{aligned}
$$

and $A\left(N_{a}, N_{b} ; t\right)$ is given by the equation

$$
\begin{aligned}
\dot{A}\left(N_{a}, N_{b} ; t\right)= & -\frac{1}{2} \sum_{\mu=a, b} N_{\mu}^{2} g_{\mu \mu} \int d \mathbf{r}\left|\phi_{\mu}(\mathbf{r}, t)\right|^{4} \\
& -N_{a} N_{b} \int d \mathbf{r}\left|\phi_{a}(\mathbf{r}, t)\right|^{2}\left|\phi_{b}(\mathbf{r}, t)\right|^{2}
\end{aligned}
$$

Using the fact that the variance of occupied states is much less than the mean $\Delta N_{a, b} \ll \bar{N}_{a, b}$, one can approximate $\phi_{\mu}\left(N_{a}, N_{b} ; \mathbf{r}, t\right) \rightarrow \phi_{\mu}\left(\bar{N}_{a}, \bar{N}_{b} ; \mathbf{r}, t\right) \equiv \bar{\phi}_{\mu}(\mathbf{r}, t)$.

The conditions for the existence of a common spatial mode for the two dressed states $\bar{\phi}_{a}(\mathbf{r}, t)=\bar{\phi}_{b}(\mathbf{r}, t) \equiv \bar{\phi}(\mathbf{r}, t)$, requires that the total effective potentials are equal [16], i.e.,

$$
\bar{N}_{a} g_{a a}+\bar{N}_{b} g_{a b}=\bar{N}_{b} g_{b b}+\bar{N}_{a} g_{a b} \equiv N g_{e f f},
$$

which implies that

$$
\frac{\bar{N}_{a}}{\bar{N}_{b}}=\frac{g_{a a}-g_{a b}}{g_{b b}-g_{a b}} .
$$

For this result to have physical solutions, it is necessary that either

$$
g_{a b}<g_{a a}, g_{b b}
$$

or

$$
g_{a b}>g_{a a}, g_{b b} .
$$

The linearized stability analysis of Ref. [16] indicates that the former case is stable, whereas the latter is unstable to a demixing instability of the condensates. We note that in our case $g_{a a}=g_{b b}=\bar{g}$, so that the average particle number in each dressed state is equal $\bar{N}_{a}=\bar{N}_{b}$. The condition for stability of the common mode $g_{a b}<g_{a a}, g_{b b}$ reduces to

$$
\frac{1}{2}\left(g_{++}+g_{--}\right)<g_{+-},
$$

which is opposite to the stability criterion in the entanglement scheme of Sorenson et al. [15].

Assuming that two bare atomic states which satisfy the stability criterion have been identified, then the breathetogether solution is $\bar{\phi}_{a}(\mathbf{r}, t)=\bar{\phi}_{b}(\mathbf{r}, t) \equiv \bar{\phi}(\mathbf{r}, t)$, where $\bar{\phi}$ satisfies the equation

$$
\frac{\partial}{\partial t} \bar{\phi}(\mathbf{r}, t)=\frac{1}{i \hbar}\left(-\frac{\hbar^{2}}{2 m} \nabla^{2}+\bar{V}(\mathbf{r})+N g_{e f f}|\bar{\phi}(\mathbf{r}, t)|^{2}\right) \bar{\phi}(\mathbf{r}, t),
$$

with $g$, the effective scattering coefficient, given by

$$
g_{\text {eff }}=\frac{3}{8}\left(g_{++}+g_{--}\right)+\frac{1}{4} g_{+-} .
$$




\section{APPENDIX B: MEAN COLLISION ENERGY IN BARE AND DRESSED CONDENSATES}

A straightforward calculation shows that the mean collision energy in a pure Fock state $\left|N_{a}: \phi_{a} ; N_{b}: \phi_{b}\right\rangle$, with definite occupancies of the dressed states $|a\rangle$ and $|b\rangle$, is given by

$$
\begin{aligned}
E_{M F}^{d}= & \frac{1}{2} \bar{g} \sum_{\mu=a, b} \frac{N_{\mu}-1}{N_{\mu}} \int d^{3} r \rho_{\mu}^{2}(\mathbf{r})+\frac{1}{2}\left(g_{++}+g_{--}\right) \\
& \times \int d^{3} r \rho_{a}(\mathbf{r}) \rho_{b}(\mathbf{r}),
\end{aligned}
$$

where $\rho_{\mu}(\mathbf{r}) \equiv N_{\mu}\left|\phi_{\mu}(\mathbf{r})\right|^{2}$ is the spatial density of dressed state $\mu=a, b$. The last result may be contrasted with the mean-field energy of two condensates in a Fock state
$\left|N_{+}: \phi_{+} ; N_{-}: \phi_{-}\right\rangle$, with definite occupancies of the bare atomic states $|+\rangle$ and $|-\rangle$,

$$
\begin{aligned}
E_{M F}^{b}= & \frac{1}{2} \sum_{\mu=+,-} g_{\mu \mu} \frac{N_{\mu}-1}{N_{\mu}} \int d^{3} r \rho_{\mu}^{2}(\mathbf{r})+g_{+-} \\
& \times \int d^{3} r \rho_{+}(\mathbf{r}) \rho_{-}(\mathbf{r}) .
\end{aligned}
$$

Comparison of these results indicates that the interstate scattering length $g_{+-}$plays a completely different role for a mixture of bare condensates and a mixture of dressed condensates. In the former it contributes to the interaction energy in proportion to the overlap of the condensate density profiles, whereas for the latter it influences only the selfinteraction energy of each dressed state component.
[1] M. Kitagawa and M. Ueda, Phys. Rev. A 47, 5138 (1993).

[2] D.J. Wineland, J.J. Bollinger, W.M. Itano, F.L. Moore, and D.J. Heinzen, Phys. Rev. A 46, R6797 (1992).

[3] D.J. Wineland, J.J. Bollinger, W.M. Itano, and D.J. Heinzen, Phys. Rev. A 50, 67 (1994).

[4] V. Meyer, M.A. Rowe, D. Kielpinski, C.A. Sackett, W.M. Itano, C. Monroe, and D.J. Wineland, Phys. Rev. Lett. 86, 5870 (2001).

[5] A. Kuzmich, K. Molmer, and E.S. Polzik, Phys. Rev. Lett. 79, 4782 (1997).

[6] K. Molmer, Eur. Phys. J. D 5, 301 (1999).

[7] K. Molmer and A. Sorensen, Phys. Rev. Lett. 82, 1835 (1999).

[8] A. Sorensen and K. Molmer, Phys. Rev. Lett. 83, 2274 (1999).

[9] A. Kuzmich, N. Bigelow, and L. Mandel, Europhys. Lett. 42, 481 (1998).

[10] J. Hald and E.S. Polzik, J. Opt. B: Quantum Semiclassical Opt. 3, S83 (2001).

[11] K. Helmerson and L. You, Phys. Rev. Lett. 87, 170402 (2001).

[12] J. Hald, J.L. Sorensen, C. Schori, and E.S. Polzik, Phys. Rev. Lett. 83, 1319 (1999).

[13] A. Kuzmich, L. Mandel, J. Janis, Y.E. Young, R. Ejnisman, and N.P. Bigelow, Phys. Rev. A 60, 2346 (1999).

[14] A. Kuzmich, L. Mandel, and N.P. Bigelow, Phys. Rev. Lett. 85, 1594 (2000).
[15] A. Sorensen, L.-M. Duan, J.I. Cirac, and P. Zoller, Nature (London) 409, 63 (2001).

[16] A. Sinatra and Y. Castin, Eur. Phys. J. D 8, 319 (2000).

[17] M.R. Matthews, B.P. Anderson, P.C. Haljan, D.S. Hall, M.J. Holland, J.E. Williams, C.E. Wieman, and E.A. Cornell, Phys. Rev. Lett. 83, 3358 (1999).

[18] J.E. Williams and M.J. Holland, Nature (London) 401, 568 (1999).

[19] M.R. Matthews, B.P. Anderson, P.C. Haljan, D.S. Hall, C.E. Wieman, and E.A. Cornell, Phys. Rev. Lett. 83, 2498 (1999).

[20] P.B. Blakie, R.J. Ballagh, and C.W. Gardiner, J. Opt. B: Quantum Semiclassical Opt. 1, 378 (1999).

[21] M.D. Barrett, J.A. Sauer, and M.S. Chapman, Phys. Rev. Lett. 87, 010404 (2001).

[22] J.E. Williams, R. Walser, J. Cooper, E.A. Cornell, and M.J. Holland, Phys. Rev. A 61, 033612 (2000).

[23] C.W. Gardiner, Phys. Rev. A 56, 1414 (1997).

[24] Y. Castin and R. Dum, Phys. Rev. A 57, 3008 (1998).

[25] F. Dalfovo, S. Giorgini, L.P. Pitaevskii, and S. Stringari, Rev. Mod. Phys. 71, 463 (1999).

[26] For the calculations performed in this paper, it is convenient to write the eigenstates of $\hat{\sigma}_{x}$ in terms of $\hat{\sigma}_{z}$ eigenstates, $|+\rangle$ and $|-\rangle$, using a nonstandard normalization, i.e., $|x+\rangle=(|+\rangle+$ $|-\rangle) / \sqrt{2}$ and $|x-\rangle=-i(|+\rangle-|-|\rangle) \sqrt{2}$. 